ARTICLE

\title{
Monte Carlo Simulation of Neutrons, Protons, Ions and Alpha Particles Involved in Soft Errors in Advanced Memories
}

\author{
Frédéric WROBEL* and Frédéric SAIGNE \\ Université Montpellier 2, Institut d’Électronique du Sud, UMR-CNRS 5214, Montpellier, FRANCE
}

\begin{abstract}
Due to cosmic rays, electronic devices such as SRAM may undergo some dysfunctions such as single event upsets also called Soft errors. A crucial issue for aerospace applications is to be able to predict the Soft Error rate of a given device in a given environment. In this work, we present a predictive tool which deals with protons and heavy ions for space applications and with neutrons for atmospheric applications. Moreover, it deals with alpha particles which can be emitted naturally within the device, during nuclear decay of polluting uranium, thorium and their daughter nuclei. Natural alpha emitters from materials, such as hafnium, are also considered. Thus, the novelty of the code is its ability to address the issues of space, avionic and ground level applications. The Monte Carlo approach allows calculating simply the single event upset and multiple bit upset cross sections as well as the associated Soft Error rates. To state whether an error occurs or not, the code is able to consider well known criterions such as the critical energy deposited in a sensitive volume or the ambipolar diffusion model.
\end{abstract}

KEYWORDS: soft error, single event upset, multiple bit upset, neutron, proton, alpha-emitters, recoil nucleus, SRAM, predictive tool, DHORIN, MC-ORACLE

\section{Introduction}

Due to the stars, protons are present in Space. ${ }^{1)}$ These protons interact with atmosphere nuclei and produce secondary particles, mainly neutrons. ${ }^{2)}$ Protons (in space) and neutrons (in atmosphere) represent a major reliability concern for microelectronic applications, especially for the aerospace community. ${ }^{3,4)}$ Nucleons are yet involved in nuclear reactions with the material nuclei of electronic device and secondary ions are then able to deposit part of their energies by creating some electron-hole pairs. Depending on the location of the nuclear reaction in the device but also on the amount of deposited energy, the produced electron-hole pairs modify the electrical behavior of the component. In SRAM for example Soft Error may be cause by a unique particle. In order to predict the reliability of a device during a given mission, predictive codes are developed. In the case of Soft Errors, the goal is to calculate either the Soft Error cross section or directly the Soft Error rate. The main difficulty of such codes is that they deal with various fields of physics: radiation environment, nuclear physics, physics of semiconductor and microelectronic.

In this work, we propose a Monte-Carlo method able to deal with particles which are at play in Soft Errors: protons and ions from Space, neutrons in atmosphere and alpha particle from natural radioactivity. The code uses pre-computed database especially for nuclear reactions which are CPU time consuming.

*Corresponding author, E-mail: frederic.wrobel@um2.fr

(c) 2011 Atomic Energy Society of Japan, All Rights Reserved.

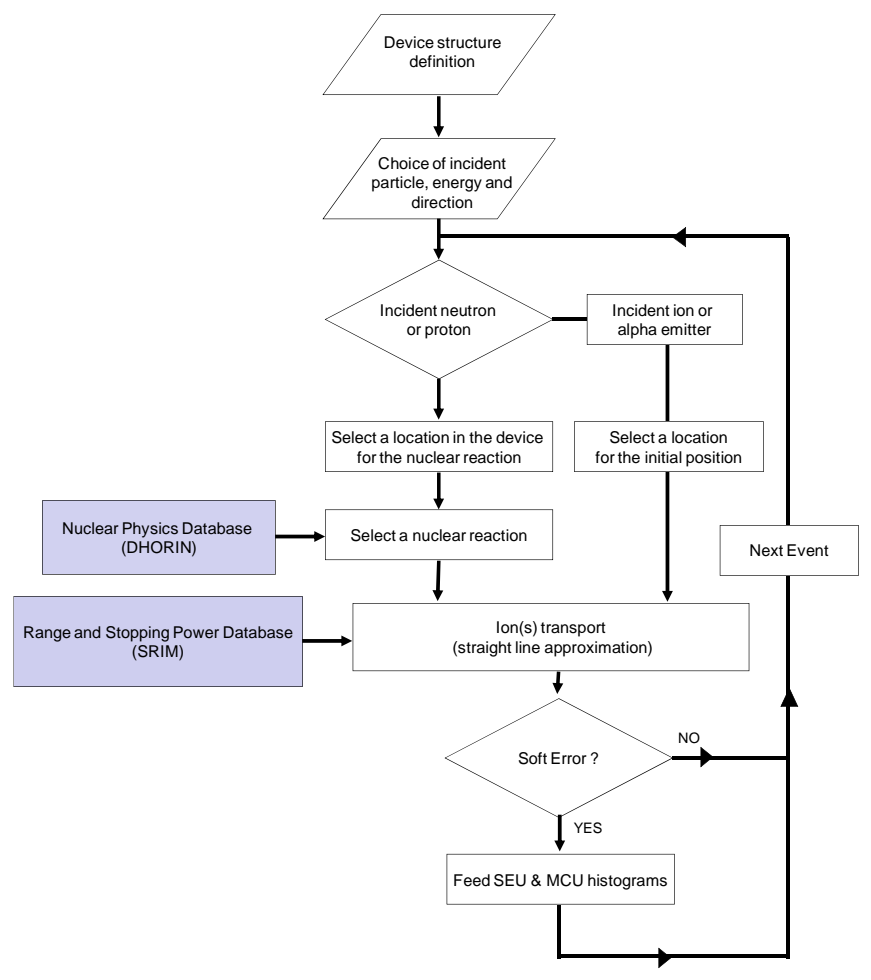

Fig. 1 Flowchart of our predictive code MC-ORACLE

\section{General Method Overview}

\section{MC-ORACLE Flowchart}

The flowchart of our predictive tool, called MC-ORACLE, is given in Fig. 1.

First of all, the device is defined in a 'universe' in which we define several volumes with three possible shapes that 
are parallelepipeds, cylinders and spheres. We then choose a radiation source which can be either neutrons, or protons or ions or alpha-emitters. Protons are not considered as ions since they mainly cause Soft Errors by nuclear reactions. On the contrary, ions induce Soft Errors mainly by direct ionization of the medium. Consequently, we did not implement nuclear processes for ion-ion interactions. The definition of the source also includes the energy of the incident particles and their directions. Notice that for alpha-emitters, the direction is automatically chosen in an isotropic distribution and their energies are obtained from a simple calculation of the Q-value. Then a location is chosen randomly in the device. For nucleons, it represents the nuclear reaction location which is determined with the mean free paths in the different materials. For incident ions, it represents the entry point of the ion in the device and is simply obtained by geometrical consideration. For alpha emitters, it corresponds to the disintegration location which is randomly chosen in a uniform distribution in the polluted volumes.

A dedicated step is then required for incident nucleons which trigger nuclear reactions. Nature, energy and direction of each secondary ion produced during nuclear reactions are determined by using a pre-calculated nuclear database built with the DHORIN code (see Section III). ${ }^{5)}$ Secondary neutral particles are not accounted for since their probability to induce a Soft Error is negligible. Because the probability for a nuclear reaction is weak we implemented the possibility to force the nuclear reaction of each primary particle. Obviously, the event is then weighted by the probability of interaction of the considered particle in the structure that is by accounting for the different material layers that can be crossed.

After a nuclear reaction, the secondary particles are transported in the structure and can only interact by ionization. This is performed by using stopping powers and ranges of ions which have previously been tabulated in a file with the SRIM code. ${ }^{6)}$ Depending on the ions properties it is possible to state whether a Soft Error occurs or not.

The Monte-Carlo method is used to simulate hundreds of thousands of primary particles in the structure. Finally error cross sections (single and multiple) or Soft Error rate can be easily determined.

\section{Soft Error Triggering Criterion}

A key part of Soft Error prediction is the choice of a criterion which determines the conditions in which an error may occur.

A widely used criterion is that of deposited energy in a sensitive volume: an error occurs when enough energy is deposited in a given sensitive region of the device. The minimum of energy deposition is called critical energy. Both sensitive region and critical energy may be determined by TCAD simulations. ${ }^{7)}$ Notice that the shape of the sensitive volume is often assumed to be a rectangular parallelepiped, assumption which is known as RPP model. ${ }^{8)}$ Sensitive volumes are defined in the structure in the RPP assumption. When an ion crosses one of the sensitive volumes, we calculate the energy deposited inside and we store the value in a histogram allowing considering the number of Soft Errors as a function of critical energy. If several sensitive volumes are crossed by one or several ions, it feeds another histogram with the second lowest energy deposited in a sensitive volume. This histogram is useful for considering multiple errors called Multiple Cell Upset (MCU).

A refinement of the RPP model can be used by considering that the boundaries of the sensitive volume are not frank but diffused. Numerically, this is accounted for by using a weighting factor which decreases with the distance to the center of the sensitive volume.

Another approach is also used in the literature to account for the diffusion of charge carriers. ${ }^{9-11)}$ The starting point is that electron-hole pairs produced during ionization are able to diffuse in the structure. As the electrical field is often low enough, electrons and holes diffuse conjointly. ${ }^{9-11)}$ This is known as the ambipolar diffusion. Diffusion laws equations are well known and allow determining the carriers' densities in the vicinity of the sensitive electrodes. It is then possible to determine the transient current pulse. These currents may be used as an input of SPICE which simulates the perturbation of the circuit and state whether an error occurs or not. Despite this criterion has a stronger physical basis, it is peculiarly time consuming.

\section{Nuclear Reactions Induced by Nucleons}

\section{Monte Carlo Simulations with DHORIN}

A crucial input for predictive tools is the history of each nuclear reaction that occurs in the device. Initially, the idea was to include a Monte-Carlo subroutine in the predictive tool for this purpose. However, simulating nuclear reactions may be time consuming and optimization can be done. For example, simulations of two similar electronic devices require calculating twice some nuclear quantities. For this reason, we chose to treat separately the nuclear reactions part and the semiconductor part. To do so, we decided to build a history of nuclear reactions which gives natures, energies and directions of secondary ions. Such database has to be computed for various materials able to be used in the microelectronic industry.

We have then developed a dedicated code, DHORIN, which stands for Detailed History Of Recoiling Ions induced by Nucleons. ${ }^{5)}$ The energy range of interest is $100 \mathrm{keV}-200 \mathrm{MeV}$ because atmospheric neutrons are very abundant in this energy range. ${ }^{12)}$ The physics of DHORIN is mainly based on the 2D-exciton model, ${ }^{13)}$ the Hauser-Feshbach formulation ${ }^{14)}$ and the generalized evaporation model. ${ }^{15)}$ The main advantage of this code is that it is able to calculate all the needed quantities (cross sections, level densities and transmission coefficients) for all nucleus that have to be treated during a reaction. For electronic application, we build a specific output which is a history of triggered nuclear reactions. For each nuclear reaction of the database, we store the nature, the energy and the direction of each secondary particle. The method thus allows accounting for the correlation between ejectiles.

Figure 2 represents the flowchart of the DHORIN code. The code starts by calling ECIS ${ }^{16)}$ in order to determine elastic and non-elastic cross sections as well as the elastic differential angular cross section, from which the differential 


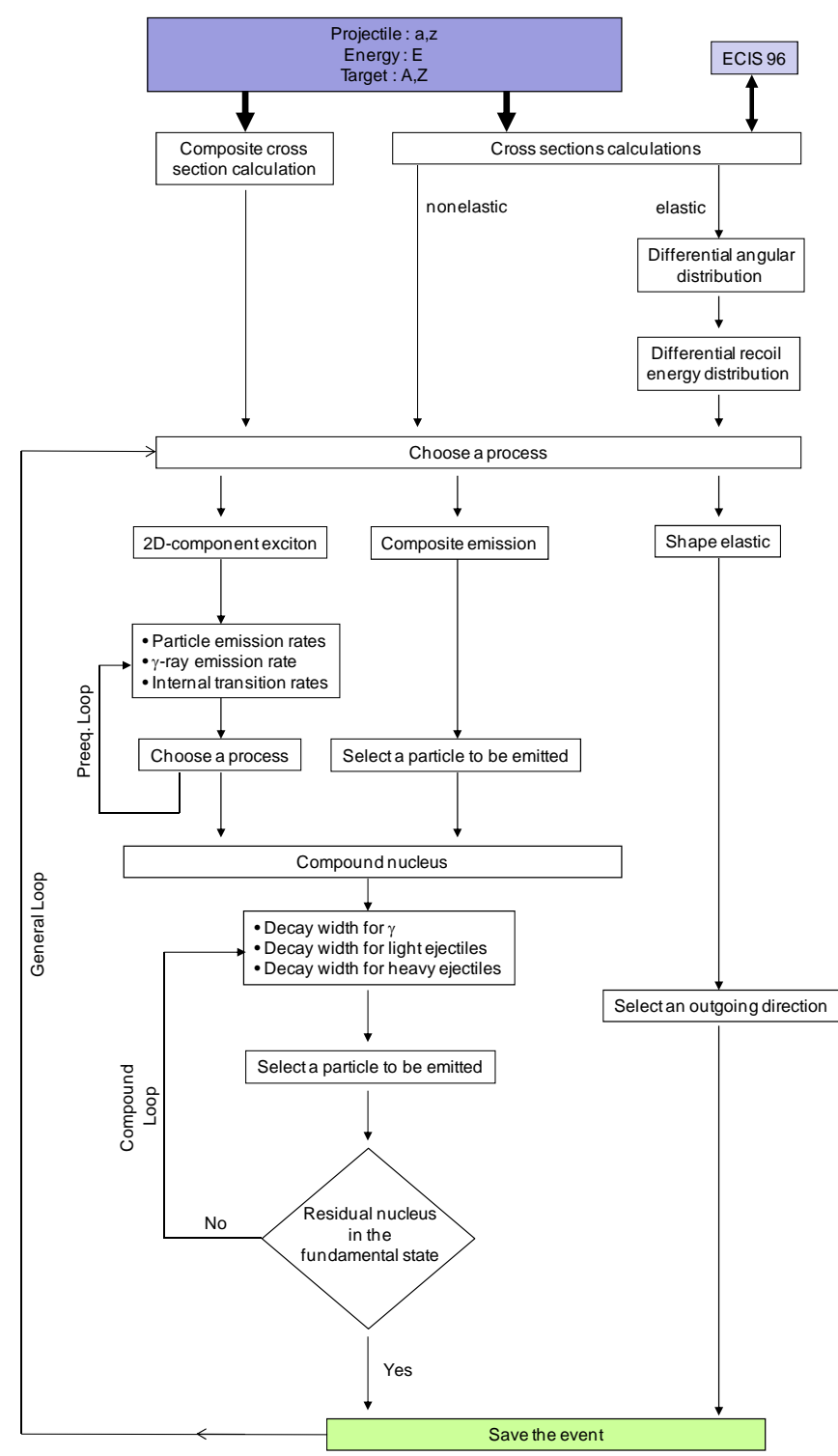

Fig. 2 Flowchart of the DHORIN code which deals with nucleon induced nuclear reactions

recoil energy cross section is computed. Non elastic reactions are treated with a Monte Carlo approach. A non-elastic reaction can start either by an exciton stage or by a composite emission (stripping, pick-up and knock-out reactions). The Monte Carlo procedure deals automatically with multiple emissions during pre-equilibrium.

After the exciton stage or the composite emission, the nucleus is considered to be a compound nucleus, which starts the equilibrium stage. Total decay widths for gammas, light ejectiles and heavy ejectiles are calculated. With respect to these decay widths a decay kind is chosen randomly as well as the outgoing particle energy. The remaining nucleus is a compound nucleus, which can also undergo ejectile emission (see "Compound. Loop" in Fig. 2). The equilibrium stage ends when the nucleus reaches its ground state.

Usually, Monte-Carlo codes treat nuclear reactions one by one but DHORIN works with a population of nuclear reactions in order to avoid a calculation that has already been done. DHORIN has been shown to be faster than a conven-

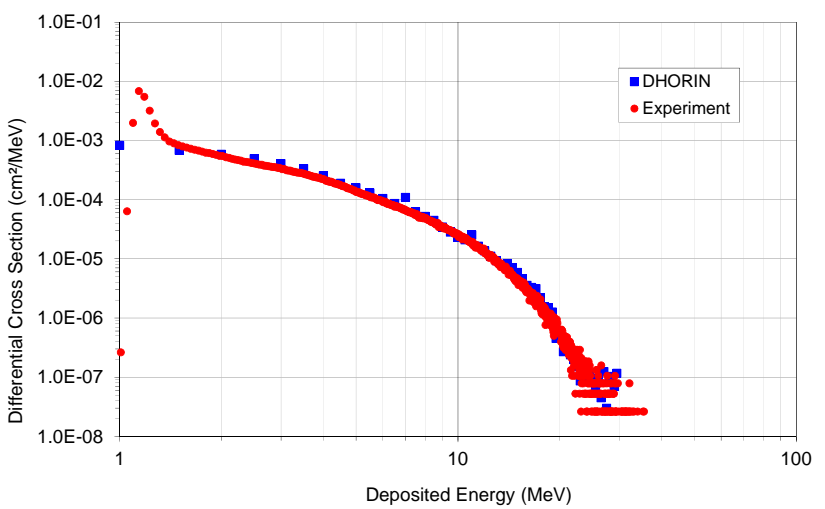

Fig. 3 Differential cross section of neutron induced energy deposition in a Silicon Diode at $30 \mathrm{MeV}$

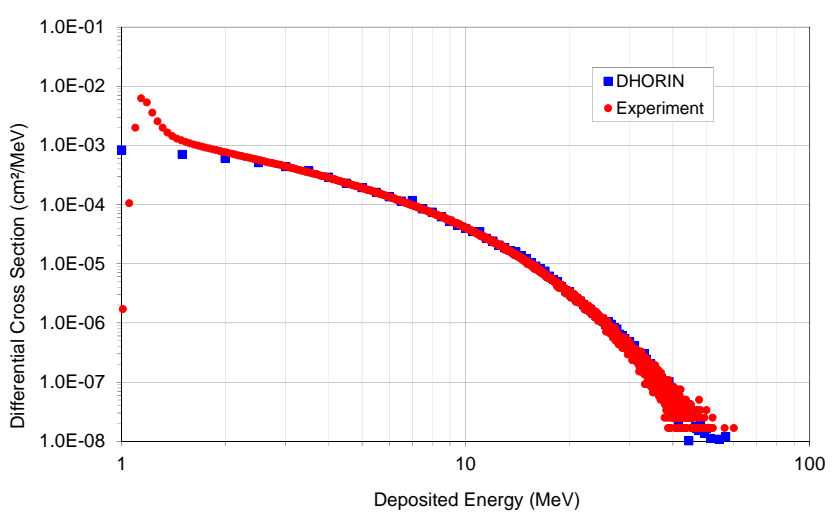

Fig. 4 Differential cross section of neutron induced energy deposition in a Silicon Diode at $63 \mathrm{MeV}$

tional Monte Carlo code by a factor of 100 . For example, for neutron induced nuclear reactions on silicon at $20 \mathrm{MeV}$, the calculation time for DHORIN is around $1 \mathrm{~s}$ for 1,000 nuclear reactions. MC-ORACLE could then use DHORIN directly as a subroutine and could run in a reasonable amount of time. However, we adopted another strategy in order to decrease the calculation time. Yet, DHORIN results are pre-computed for various energies and materials and are used in MC-ORACLE as a simple input. Finally, MC-ORACLE is able to simulate 100,000 nuclear reactions in a device within a few seconds.

\section{Experimental Validation}

Calculated excitation functions with DHORIN for silicon and aluminum are shown to be in good agreement with the EXFOR database. ${ }^{5)}$ Notice that silicon has been previously widely investigated since it is the most abundant material in microelectronic devices.

Moreover, we have performed experiments at UCL by irradiating a silicon diode with neutrons at 30 and $63 \mathrm{MeV}$ at the CYCLONE facility in UCL. ${ }^{17)}$ Figures 3 and $\mathbf{4}$ plot the obtained cross sections as a function of deposited energy. Also plotted are the results of our MC-ORACLE simulations. Hundreds of thousands of nuclear reactions (extracted from DHORIN) are simulated in the diode. For each nuclear reaction, the secondary ions are transported in the diode and their energy deposition is calculated. Results are in very good agreement and contribute to the validation of DHORIN and 
MC-ORACLE. Notice that the observe discrepancy observed at low energy (below $1.5 \mathrm{MeV}$ ) is experimentally attributed to the noise induced by electronics.

\section{Alpha Emitters}

Neutrons, protons and heavy ions are the source of a radiation environment in which electronic devices are present. These radiations are a consequence of cosmic rays and represent an extrinsic radiation environment.

There is also an intrinsic source of reliability degradation which comes from the alpha emitters introduced into the device. These radioactive nuclei are either radioactive materials or radioactive impurities. The first case corresponds to materials that naturally contain a portion, even small, of alpha emitters. Hafnium for example, which already replaces silicon in gate oxide, is naturally composed of $0.162 \%$ of the alpha emitter isotope ${ }^{174} \mathrm{Hf}^{18)}$

The second case, the impurities, corresponds to a pollution of uranium or thorium elements and their daughter nuclei. These elements are present in the natural environment and unavoidably pollute materials used during device process.

As alpha particles have been shown to be a major contribution to Soft Error at ground level, we implemented a subroutine which deals with their disintegrations.

Because alpha emissivity of materials used in microelectronics can be measured, ${ }^{19)}$ we can deduce the concentration of alpha pollutants in the device. ${ }^{20)}$ MC-ORACLE is able to determine this emissivity by making the assumption of secular equilibrium. Then knowing the concentration of impurities, MC-ORACLE triggers nuclear decay and follow the emitted alpha particle in the device to state whether an error occurs. Knowing the rate of disintegrations, which is given by a well-known exponential decay, it is finally possible to calculate the Soft Error rate due to alpha particle. The method has been investigated in previous work ${ }^{20)}$ and shows a good agreement with experimental data.

\section{Conclusions}

We presented a Monte Carlo approach to obtain Soft Error cross sections (simple and multiple bit) as well as the Soft Error rate when the fluence of particles during a mission is known. We implemented the method in the MC-ORACLE code which deals with neutrons, protons, ions and alpha particles from natural radioactivity. The main goal of the code is to treat various source of radiation that causes Soft Error but also to evaluate models of the triggering criterion. In the current version, the RPP is implemented for both frank and diffuse boundaries. The diffusion model is presently being implemented.

The originality of the code is to pre-compute CPU time consuming calculations such as nuclear reactions induced by nucleons. To do so we previously developed the DHORIN code which exhibits good agreement with EXFOR libraries as well as energy deposition measurements.

Another crucial approximation is that of considering that ions move in straight line. This allows reducing calculating time and it is possible to simply use the SRIM code to obtain stopping power.

Finally, the code is consistent and is a useful tool for evaluating the reliability of modern devices. We planned to implement a subroutine able to deal with single event transient in order to treat other devices.

\section{References}

1) G. Gloeckler, "Composition of Energetic Particle Populations in interplanetary Space," Rev. Geophys. Space Phys. 17, 569 (1979).

2) W. N. Hess, H. W. Patterson, R. Wallace, E. L. Chupp, “Cosmic-Ray Neutron Energy Spectrum,” Phys. Rev., 116, 445 (1959).

3) J. F. Ziegler, G. R. Srinivasan, IBM J. Re. Develop., 40[1], 19-39 (1996).

4) E. Normand, "Single Event effects in Avionics," IEEE Trans. Nucl. Sci., 43, 2742-2750 (1996).

5) F. Wrobel, "Detailed History of Recoiling Ions induced by Nucleons,” Comput. Phys. Comm., (2007); doi :10.1016/j.cpc.2007.08.005

6) J. F. Ziegler, J. P. Biersack, U. Littmark, The Stopping and Range of Ions in Solids, Pergamon Press, New York (1985), (new edition in 2009), online http://www.srim.org/

7) J.-M. Palau, G. Hubert, K. Coulie, B. Sagnes, M. C. Calvet, S. Fourtine, "Device simulation study of the SEU sensitivity of SRAM's to internal ion tracks generated by nuclear reactions," IEEE Trans. Nucl. Sci., 48, 225-231 (2001).

8) E. L. Petersen, J. C. Pickel, E. C. Smith, R. J. Rudeck, J. R. Letaw, "Geometrical factor in SEE rate calculations," IEEE Trans. Nucl. Sci., 40, 1888-1909 (1993).

9) J.-M. Palau, F. Wrobel, K. Castellani-Coulié, M.-C Calvet, P. E. Dodd, F. W. Sexton, "Monte Carlo Exploration of Neutron-Induced SEU-Sensitive Volumes in SRAMs," IEEE Trans. Nucl. Sci., 49, 3075-3081 (2002).

10) J.-M. Palau, M.-C. Calvet, P. E. Dodd, F. W. Sexton, P. Roche, "Contribution of Device Simulation to SER Understanding," IEEE 41st International Reliability Physics Symposium, Dallas, (2003).

11) T. Merelle , H. Chabane, J.-M. Palau, K. Castellani-Coulié, F. Wrobel, F. Saigné, B. Sagnes, J. Boch, J. R. Vaille, G. Gasiot, P. Roche, M.-C. Palau, T. Carrière "Criterion for SEU occurrence in SRAM Deduced From Circuit and Device Simulations in Case of Neutron-Induced SER,” IEEE Trans. Nucl. Sci., 52, 1148-1155 (2005).

12) W. N. Hess, E. H. Canfield, R. E. Lingenfelter, "Cosmic-Ray Neutron Demography,” J. Geophys. Res., 66, 665-677 (1961).

13) J. Dobes, E. Betak, "Two-component exciton model," Zeit. Phys., A310, 329-338 (1983).

14) M. Uhl, "Cross section determination using the statistical model and including angular momentum and parity conservation,” Acta Physica. Austri., 31[3], 245-270 (1970).

15) S. Furihata, "Statistical analysis of light fragment production from medium energy proton-induced reactions," Nucl. Instr. Meth. Phys. Res., B171[3], 251-258 (2000).

16) J. Raynal, CEA Saclay Report No. CEA-N-2772 (1994).

17) S. Rocheman, F. Wrobel, J-R Vaillé, F. Saigné, C. Weulersse, N. Buard, T. Carrière, "Neutron Induced Energy Deposition in a Silicon Diode,” IEEE, Trans. Nucl. Sci., 55[6], 3146-3150 (2008).

18) F. Wrobel, J. Gasiot, F. Saigné, A. D. Touboul, "Effects of atmospheric neutrons and natural contamination on advanced microelectronic memories,” Appl. Phys. Lett., 93, 064105 (2008). 
19) JEDEC Standard, Measurement and Reporting of Alpha Particle and Terrestrial Cosmic Ray-Induced Soft Errors in Semiconductor Devices, JESD89A, (2006).
20) F. Wrobel, J. Gasiot, F. Saigné, "Hafnium and Uranium Contributions to Soft Error Rate at Ground Level," IEEE, Trans. Nucl. Sci., 55[6], 3141-3145 (2008). 